\title{
The projective invariants of four medials
}

\author{
By H. W. Tornbull. \\ (Received 16th August, 1941. Read 1st November, 1941.)

\section{Introduction}

The theory of four particular linear forms, or matrices of $k$ columns and $2 k$ rows, occurred to me many years ago in an attempt to study the invariants of any number of compound linear forms, or subspaces within a space of $n$ dimensions. In what follows, the invariant theory is given, and its significance for a study of the general matrix of $k$ rows and columns is suggested. The collineation used in $\S 4$ was considered by Mr J. H. Grace ${ }^{1}$, who emphasized the importance of the $k$ cross ratios upon transversal lines of four [ $k-1$ ]'s in $[2 k-1]$. It seemed appropriate to examine these cross ratios which are irrational invariants $\mu_{i}$ of the figure of four such spaces, and to work out their relation to the known rational invariants $X_{i}$. The main result is given in $\S 5(7)$. In $\S 5(10)$ it is shewn that the harmonic section of a line transversal of the four spaces exists when a linear relation holds between the invariants.

This work is supplementary to a geometrical treatment ${ }^{2}$ of matrix pencils by means of the intersection of one $[k-1]$ and a certain locus $V_{k}^{k}$ described by all line transversals of the other three $[k-1]$ 's.

The projective invariants of four medials

$\S 1$. By a medial is meant a linear space $[k-1]$ in the space $[2 k-1]$; so that, on taking $k=1,2,3$ etc. in succession, a point is a medial on a straight line, a straight line is a medial in three dimensions, a plane is a medial in five dimensions, and so on. Within the hierarchy of subordinate linear spaces, ranging from point [0] to prime $[2 k-2]$, a medial stands midway, and is self dual. It is characteristic of odddimensional space.

Analytically a medial is defined, with reference to a simplex of $2 k$ points $\{1,0, \ldots, 0\},\{0,1,0 \ldots, 0\}$, etc. in $2 k$ homogeneous coordinates, by a matrix $A$ of $2 k$ rows and $k$ columns: let us say

$$
A=\left[a_{1} a_{2} \ldots a_{k}\right] \text {, }
$$

1 " Extension of a geometrical porism and other theorems," Proc. Camb. Phil. Soc., 25 (1929), 421-432.

${ }^{2}$ Phil. Trans. Royal Soc., A 239 (1942), 233-267 (No. 805). 
where each column $a_{j}$ denotes a point of the medial, and these $k$ points are linearly independent. Similarly for further medials $B, C, D$, each defined by $k$ such points $b, c$, or $d$. Let

$$
A B=\left[a_{1} a_{2} \ldots a_{k} b_{1} b_{2} \ldots b_{k}\right], \quad(A B)=\left(a_{1} \ldots a_{k} b_{1} \ldots b_{k}\right),
$$

respectively denote the matrix and determinant of $2 k$ rows and columns, arranged in the specified order. Then $(A B)$ is the only irreducible projective invariant of two medials. It vanishes if the medials have a point in common, but not otherwise. When $k=2$ it is the mutual moment of two lines.

To prove this we take any such rational integral invariant as the sum of non-zero terms $T$, each of which is a product of factors $\left(e_{1} e_{2} \ldots e_{2 k}\right)$, where each $e$ is either an $a$ or a $b$, this being an immediate consequence of the Fundamental Theorem ${ }^{1}$. The same theorem shews that the medial $A$ is given by the ground form $(A \pi)=\Sigma A_{i \ldots} \pi_{. . j}$, where $\pi$ denotes a variable medial which replaces $B$ in $(A B)$ : that is, the $k$ points $a$ are convolved in the ground form and also in every invariant (or more generally, concomitant) of the medial $A$. Similarly for $B$. Such convolution is either explicit ${ }^{2}$ or implicit in each term of $T$. But by a fundamental identity we can convolve $A$ explicitly in the first factor of each term of $T$, so accounting for $k$ of the $2 k$ places $e_{j}$. The remaining $k$ places can only belong to $B$; and since there are in all $2 k$ different columns $a$ or $b$, the factor is non-zero only when it is $(A B)$ or a mere derangement of this. This proves the result.

Three medials $A, B, C$ have no essentially new type of invariant. For if $T$ has one factor it can only be $(A B)$ or $(B C)$ or $(C A)$ : if two factors, one of the medials must be repeated and gives, say, terms $\left(A B^{\prime} C^{\prime}\right)\left(A B^{\prime \prime} C^{\prime \prime}\right)$, where $B^{\prime} B^{\prime \prime}$, and $C^{\prime} C^{\prime \prime}$ are convolutions of $B$ and $C$ respectively. By the fundamental identity $B$ can be convolved explicitly in the first factor at the expense of symbols of $A$ or $C^{\prime}$. Since the removal of any $a$ from the first to the second factor yields

1 Turnbull, Theory of Determinants, Matrices and Inrariants (Blackie, 1928), 203.

The symbol $A$ is convolved explicitly when all its $k$ partial symbols $a$ occur in one bracket factor $\left(e_{1} \ldots \ldots e_{2 k}\right)$, and implicitly when they occur distributed over two or more such factors. In the latter case the expression involves several terms $T$, the sum of which is necessarily unaltered by determinantal permutation of the partial symbols $a$ (cf. loc. cit. p. 46). 
a zero result (with duplicates $a$ in the second factor), only $C^{\prime}$ can be removed: and the term becomes $(A B)(A C)$, which reduces. For three or more factors in $T$, the same kind of result follows by convolving an $A$ in each of the first, second, .... factors until all $A$ are exhausted, and then convolving a $B$ in the last, last but one, ... factors.

Four medials $A, B, C, D$ have many new types of invariant, with remarkable properties. Only those which are linear in each medial are discussed here. Such invariants are of degree $4 k$ in the symbols $a, b, c, d$, and by the fundamental theorem must be an aggregate of terms

$$
\left(a_{j} \ldots b_{l} \ldots c_{m} \ldots d_{n} \ldots \ldots\right)\left(a_{p} \ldots b_{q} \ldots c_{r} \ldots d_{s} \ldots \ldots\right)
$$

involving exactly $k$ symbols of each kind $a, b, c, d$ within two determinantal factors of $2 k$ columns each. As before, each set $A, B, C$ or $D$ is implicitly or explicitly convolved in the aggregate. Hence one set $B$ may be explicitly convolved in the first factor, another $D$ in the second, and the invariant may be taken to be

$$
\left.\begin{array}{rl}
X_{i} & =\Sigma\left(A_{i} B_{k} C_{l}\right)\left(C_{i} D_{k} A_{l}\right), \quad k=i+l, \\
& =\left(A_{i}^{:} B_{k} C_{l}^{:}\right)\left(C_{i}^{:} D_{k} A_{l}\right)
\end{array}\right\} .
$$

Here $A_{i}=a_{1} a_{2} \ldots a_{i}$, that is the matrix of the first $i$ symbols $a, B_{k}$ denotes all the symbols $b, C_{l}=c_{i+1} c_{i+2} \ldots c_{k}$, the last $l$ symbols $c$, and so on. We have in fact partitioned both $A$ and $C$ into $i$ and $l$ columns :

$$
A=A_{k}=A_{i} A_{l}=A_{i} A_{k-i}, \quad C=C_{k}=C_{i} C_{l}=C_{i} C_{k-i} .
$$

The summation $\Sigma$ extends to $\left(\begin{array}{c}k \\ i\end{array}\right)^{2}$ terms due to separate determinantal permutation of $A$ and $C$. It is also indicated by the dots beside $A_{i}, A_{l}$ (giving $\left(\begin{array}{c}k \\ i\end{array}\right)$ terms), and the double dots beside $C_{l}, C_{i}$ (giving $\left(\begin{array}{c}k \\ i\end{array}\right)$ terms).

For example, if $k=2, i=1$,

$X_{1}=\left(a_{1} B c_{2}\right)\left(c_{1} D a_{2}\right)-\left(a_{2} B c_{2}\right)\left(c_{1} D a_{1}\right)+\left(a_{2} B c_{1}\right)\left(c_{2} D a_{1}\right)-\left(a_{1} B c_{1}\right)\left(c_{2} D a_{2}\right)$. It will be proved that, of all such $X_{i}$, due to permutations of $A, B, C, D$ and to the range of values $i$ from 0 to $k$ inclusive, exactly $l i-2$ invariants are irreducible. Within the determinant, by derangement of columns, we have

$$
A_{i} A_{l}=(-)^{i l} A_{l} A_{i}, \quad\left(A_{i} B C_{l}\right)=(-)^{i l+k}:\left(C_{l} B A_{i}\right)
$$


Hence on writing $X_{i}=(A B C D)_{i}$ and then interchanging the factors and deranging the columns we find that

$$
X_{i}=(A B C D)_{i}=(A D C B)_{l}=(C D A B)_{i}=(C B A D)_{l} .
$$

In fact a term of $(A D C B)_{l}$ is $\left(A_{l} D_{k} C_{i}\right)\left(C_{l} B_{k} A_{i}\right)$, which deranges to the original term $\left(A_{i} B_{k} C_{l}\right)\left(C_{i} D_{k} A_{l}\right)$ of (1) with an even number of changes in sign.

It is natural to continue such derangement of the letters. What happens, for example, when $A$ and $B$ are interchanged? We find that

$$
X_{i}=\sum_{j=i}^{k}(-)^{j}\left(\begin{array}{c}
j \\
i
\end{array}\right)(B A C D)_{j} \text {. }
$$

Proof. Consider the expression $\left(\dot{A}_{i} B_{k} C_{l}\right)\left(C_{i} D_{k} A_{l}\right)$, where at present $A$ alone is permuted. This consists of $\left(\begin{array}{c}k \\ i\end{array}\right)$ terms all belonging to $X_{i}$. By a fundamental identity [loc. cil. p. 45] $A$ can be convolved explicitly in the first factor; this brings all $A_{l}$ into the factor and displaces $l$ letters $b$ or $c$ in every possible different way. Suppose then that

$$
B_{k}=B_{j} B_{k-j}, \quad C_{l}=C_{j-i} C_{k-j},
$$

where $j=i, i+1, \ldots, k$ in turn. Thus $A_{l}$ displaces $k-j$ of the $b$ and $j-i$ of the $c$; and the whole operation yields the identity

$$
\left(A_{i}^{*} B_{k} C_{l}\right)\left(C_{i} D_{k} A_{l}^{\dot{j}}\right)=\sum_{j=i}^{k}\left(A_{i} B_{j}^{*} A_{l} C_{k-j}^{;}\right)\left(C_{i} D_{k} B_{k-j}^{*} C_{j-i}^{*}\right) .
$$

The right hand expression is a triple summation, indicated by the dots, the double dots and the $\Sigma$. The $C$ summation (indicated by double dots) evidently has $\left(\begin{array}{c}l \\ j-i\end{array}\right)$ terms.

Now permute all $k$ of the letters $c$ determinantally (that is, with change of sign for each interchange of two letters), so forming $k$ ! such identities, including this one; and add the results. Briefly, operate on each side of the identity with Young's negative symmetric group operator $\left\{c_{1} c_{2} \ldots c_{k}\right\}^{\prime}$. On the left we have at once

$$
l ! i !\left(A_{i}^{*} B_{k} C_{i}^{\Sigma}\right)\left(C_{i}^{*} D_{k} \dot{A_{l}}\right)
$$

which is $l ! i ! X_{i}$. On the right fix $j$ and $B$ provisionally, and consider the effect on the $\left(\begin{array}{c}l \\ j-i\end{array}\right)$ terms of the $C$ summation. The operation gives $k !\left(\begin{array}{c}l \\ j-i\end{array}\right)$ such terms. But since the operation effects all the 
$c$ 's, the terms must be identical, apart from sign, with those of

$$
\Gamma=\left(\ldots C_{k-j}^{*}\right)\left(C_{i}^{*} \ldots C_{j-i}^{*}\right)
$$

which has $\left(\begin{array}{c}k \\ j\end{array}\right)$ terms. Since all the operations on the $c$ 's are determinantal it follows that, whenever they produce the same term of $\Gamma$, they must produce it with the same sign. Again, since all the $k$ letters are permuted determinantally, then if one term of $\Gamma$ is produced, every term with its correct sign is produced. Thus the right hand member of the identity can only be a multiple of $\Gamma$, which is found by counting all the terms on the right and dividing by the number of terms in $\Gamma$. This gives

$$
k !\left(\begin{array}{c}
l \\
j-i
\end{array}\right) \div\left(\begin{array}{l}
k \\
j
\end{array}\right)=\frac{l ! j !}{(j-i) !}
$$

On dividing throughout by $l ! i$ ! and restoring the summations for $j$ and $B$ we have

$$
X_{i}=\sum_{j=i}^{k}\left(\begin{array}{l}
j \\
i
\end{array}\right)\left(A_{i} B_{j}^{*} A_{l} C_{k-j}^{:}\right)\left(C_{i}^{:} D_{k} B_{k-j}^{\cdot} C_{j-i}^{:}\right)
$$

where the double dots now indicate the permutation of $C_{k-j}$ with $C_{j}\left(=C_{i} C_{j-i}\right)^{*}$. On rearranging the matrices within the two determinants of a term we obtain the arrangement

$$
\left(B_{j} A_{i} A_{l} C_{k-j}\right)\left(C_{i} C_{j-i} D_{k} B_{k-j}\right)
$$

which involves $N^{T}=i j+(2 k-j)(j-i)$ changes of sign. Since $N \equiv-j^{2} \equiv j \bmod 2$, we now have

$$
X_{i}=\sum_{j=i}^{k}(-)^{j}\left(\begin{array}{l}
j \\
i
\end{array}\right)\left(B_{j}^{*} A_{k} C_{k-j}^{:}\right)\left(C_{j}^{:} D_{k} B_{l}^{*}\right)=\sum_{j=i}^{k}(-)^{j}\left(\begin{array}{l}
j \\
i
\end{array}\right)(B A C D)_{j},
$$

which proves the result.

On writing $(-)^{j}\left(\begin{array}{l}j \\ i\end{array}\right)=\lambda_{j}$ and combining the results (4) and (5), we have

$$
X_{i}=(A B C D)_{i}=\Sigma \lambda_{j}(B A C D)_{j}=\Sigma \lambda_{j}(C D B A)_{j}=(D C B A)_{i}=(B A D C)_{i} .
$$

Hence we may interchange any pair of $A, B, C, D$, together with the complementary pair, without altering the value of $X_{i}$. This means 
that the 24 permutations of $A, B, C, D$ fall into three groups of eight, $X, Y$ and $Z$ say, of which the $X$ group is

$$
\begin{aligned}
X_{i} & =(A B C D)_{i}=(B A D C)_{i}=(C D A B)_{i}=(D C B A)_{i} \\
& =(A D C B)_{l}=(B C D A)_{l}=(C B A D)_{l}=(D A B C)_{l}
\end{aligned}
$$

where $i+l=k$. We may characterize these by writing $X_{i}=$ $\{A C, B D\}_{i}$, the pair $A C$ always separating the pair $B D$. Furthermore let $Y_{i}=(A D B C)_{i}, Z_{i}=(A C D B)_{i}$. Then

$$
X_{i}=\{A C, B D\}_{i}, \quad Y_{i}=\{A B, D C\}_{i}, \quad Z_{i}=\{A D, C B\}_{i} .
$$

Relation (5) expresses $X_{i}$ linearly in terms of the set $Z$. By (6) we can interchange $B$ with $D$ and $i$ with $l$, getting a new form of (5),

$$
X_{i}=\sum_{j=l}^{k}(-)^{j}\left(\begin{array}{l}
j \\
l
\end{array}\right)(D A C B)_{j}
$$

which gives $X_{i}$ linearly in terms of the set $Y$. Hence (5) and (8) give the result

$$
X_{i}=\sum_{j=i}^{k}(-)^{j}\left(\begin{array}{l}
j \\
i
\end{array}\right) Z_{k-j}, \quad X_{i}=\sum_{j=l}^{k}(-)^{j}\left(\begin{array}{l}
j \\
l
\end{array}\right) Y_{j} .
$$

These can conveniently be written in matrix notation as $X=P Z$, $X=Q Y$, where $X=\left\{X_{0}, X_{1}, \ldots, X_{k}\right\}, Y, Z$ are three column vectors, and $P, Q$ are $(k+1) \times(k+1)$ matrices. By cyclic interchange, from (7), we have at once

$$
X=Q Y, \quad Y=Q Z, \quad Z=Q X, \quad X=P Z, \quad Y=P X, \quad Z=P Y,
$$

so that $P^{3}=Q^{3}=I$ the unit matrix, and $P=Q^{-1} .^{1}$

Another form of the relations between the sets $X, Y, Z$ is obtained by taking an arbitrary. variable $\mu$ and

$$
\mu^{\prime}=1 /(1-\mu), \quad \mu^{\prime \prime}=1-1 / \mu .
$$

It is readily verified by (9) that

$$
\begin{array}{rlrl} 
& X_{0}-X_{1} \mu+X_{2} \mu^{2}-\ldots+(-)^{k} X_{k} \mu^{k} \\
= & \left(Y_{0}-Y_{1} \mu^{\prime \prime}+\ldots\right. & & \left.+(-)^{k} Y_{k} \mu^{\prime k}\right)(-\mu)^{k} \\
= & \left(Z_{0}-Z_{1} \mu^{\prime}+\ldots\right. & & \left.+(-)^{k} Z_{k} \mu^{\prime k}\right)(\mu-1)^{k}
\end{array}
$$

for all values of $\mu$. The close analogy with the theory of cross ratios is explained below (p. 67).

1 This matrix, $P$ of triangular form with binomial coefticients as elements, was studied in the Journal London Math. Soc., 2 (1927), 242-4. Cf. Vaidyanathaswamy, "Integer roots of the unit matrix," Jowrnal London Mfarh. Soc., 3 (1928), 121-4. 


\section{Irreducibility of the Invariants}

§2. Theorem. Exactly $k-2$ of the invariants $X_{i}, Y_{i}, Z_{i}$ are irreducible. Proof. If $i=0, i=k$ we have the forms

$$
X_{0}=(B C)(D A), \quad X_{k}=(A B)(C D)
$$

which are obviously reducible. Hence all six $X_{0}, \ldots, Z_{k}$, of suffix 0 or $k$, reduce at once. Moreover each $Y_{i}$ and $Z_{i}$ is reducible to a sum of the $X_{i}$; also $X_{0}-X_{1}+X_{2}-\ldots=Y_{0}$. Hence at most $k-2$ of the whole set are irreducible; $X_{1}, X_{2}, \ldots, X_{k-2}$, say.

Nor can any further relation $\sum_{h=0}^{k} \lambda_{h} X_{h}=0$ hold between the $\boldsymbol{X}_{i}$, where the $\lambda_{h}$ are numerical and not all zero. For if $\lambda_{i} \neq 0, i>1$, then we consider the special case for which

$$
B_{k}=A_{l} C_{i}, \quad D_{k}=A_{i} C_{l}
$$

In this case, whenever $h>i$, every term of $X_{h}$, as given by the series $\$ 1$ (1), will contain more than $k$ of the $a$ 's in the first factor, and therefore at least two equal $a$ 's. This causes $X_{h}$ to vanish. Likewise the second factor causes $X_{h}$ to vanish whenever $h<i$. Hence the assumed identity $\sum_{h=0}^{k} \lambda_{h} X_{h}=0$ reduces to the single term $\lambda_{i} X_{i}$, with $h=i$ only, which is $\lambda_{i}\left(A_{i}^{*} A_{l} C_{i} C_{l}^{i}\right)\left(C_{i}^{:} A_{i} C_{l} A_{i}\right)$. But this is equal to $\lambda_{i}(A C)^{2}$ which does not vanish identically. This contradicts the assumption that a relation $\Sigma \lambda_{i} X_{i}=0$ exists, and proves the theorem.

\section{Matrix Outer Products}

§3. Consider the covariant $(x P Q R)=\left(x P Q^{\prime *}\right)\left(Q^{\prime \prime} R\right)$ of three spaces $P, Q, R$ and a point $x$, all in [n-1]. Let the four matrices $x, P, Q, R$ have $n$ rows, but $1, p, q, r$ columns respectively, where $1+p+q^{\prime}=q^{\prime \prime}+r=n, \quad q=q^{\prime}+q^{\prime \prime}$.

Equated to zero this covariant, being linear in $x$, means that the point $x$ lies in a certain prime $\pi$. Evidently each point of $P$ belongs to $\pi$, since $x P$ vanishes when $x$ is in $P$. Furthermore by convolving $Q$ in the first factor we have

$$
(x P Q R)=\Sigma(Q x \ldots)(\ldots R)+\Sigma(Q \ldots)(\ldots x R)
$$

where the first summation represents a prime containing $Q$, and the second, one containing $R$.

Hence $(x P Q R)$ represents the prime through $P$ and the inter- 
section of $Q$ and $R$. So too does $(x P S)$ where $S$ is this intersection, which is a $\left[q^{\prime}-1\right]$. Hence we can write

$$
Q R=Q^{\prime \cdot}\left(Q^{\prime \prime} R\right)=S .
$$

Equally well $S=(-)^{q r} R^{\prime}\left(R^{\prime \prime} Q\right)$, by convolving $Q$ in the last factor. Such products $Q R, R Q$ are virtually the regressive outer products of Grassmann ${ }^{1}$. They express the intersection $\left[q^{\prime}-1\right]$ of $Q$ and $R$ as a linear combination either of the $\left(\begin{array}{c}q \\ q^{\prime}\end{array}\right)$ spaces such as $Q^{\prime}$, or of the $\left(\begin{array}{c}r \\ q^{\prime}\end{array}\right)$ spaces $R^{\prime}$.

When $P \ldots Q R$ is a set of several such matrices, of $n$ rows and $p+\ldots+q+r$ columns, let the columns in this order be partitioned into determinants (each of $n$ rows and columns) from the right, with a residue of $\sigma$ initial columns $(0 \leqq \sigma<n)$. Also let each matrix $P$ etc., which happens to be broken by this process, undergo separate determinantal permutation.

We shall call the resulting expression a matrix outer product (of currency $\sigma$ and weight $w$, where $w$ is the number of determinantal factors).

For example

$$
B_{k} C_{k} D_{k} A_{l}=B_{k} C_{i}^{*}\left(C_{i}^{\bullet} D_{k} A_{l}\right)^{\bullet}
$$

is a matrix outer product of four factors as shewn. Here $\sigma=k+l$, $w=1, n=2 k$. When $\sigma=0$ the product is an invariant: for example

$$
A_{i} B_{k} C_{k} D_{k} A_{l}=\left(A_{i} B_{k} C_{l}^{*}\right)\left(C_{i}^{*} D_{k} A_{l}\right)
$$

is the outer product (and an invariant) of five spaces. But $X_{i}$, which involves derangement of the two spaces $A_{i}, A_{l}$ also, is not an outer product.

When $\sigma>0$ the outer product, if non-zero, is always a space $[\sigma-1]$, as in the example $Q R$ above. For each factor in turn from the right can if necessary be merged into a simpler space by substitutions of type (1). Thus, in (2), $C_{k} D_{k} A_{l}$ denotes the intersection $L$, an $[l-1]$, of $C$ with the space $D A$, while the whole product denotes the $[k+l-1]$ space $B L$. The method applies to any such case.

Partitioning the product $P \ldots Q Q R$ from right to left would equally well lead to a space $[\sigma-1]$; but in general it would be a different space.

The case when $\sigma=1$ is of special importance. Then the product

1 Cf. Grassmann, Ausdehnungslehre (1862), 113 p. 83 . Forder, Calculus of Estension (Cambridge 1941), 217-249. 
determines a point of $P$. Consider, for example, the product $P Q R$ in four dimensions where

$$
P=a b, Q=c d, R=e f
$$

are three lines through pairs of points $a, b, c, d, e, f$. We have by definition

$$
P Q R=a b . c d . e f=a(b c d e f)-b(a c d e f),
$$

which is manifestly a point on the line $a b$, the point $\lambda a+\mu b$ in fact, where the numerical coefficients are

$$
\lambda=(\text { bcdef }), \mu=(\text { cadef }) .
$$

But the fundamental identity $a^{\bullet}\left(b^{*} c^{*} d^{\bullet} e^{\bullet} f^{\bullet}\right)=0$, of six terms, at once gives

$$
P Q R+Q R P+R P Q=0,
$$

where $Q R P$ is a point $c^{*}\left(d^{*} a b e f\right)$ of the line $c d$, and $R P Q$ is a point of ef. Hence the identity, which shews that these three points are in line, gives the three collinear points on the transversal line of three lines in [4].

Similarly for any such $P Q R$ for which $\sigma=1$. The corresponding identity of $n+1$ terms renders the three points $P Q R, Q R P, R P Q$ collinear. That is, the combination $P Q R$ represents the line transversal of three such spaces $P, Q, R$ in $[n-1]$, while the permutation denotes the point of intersection of the line and the space first named.

Four factors $P Q R S$, with $\sigma=1$, would give the four points of a transversal plane, by the corresponding identity. And so on.

\section{The Point Collineation of Four Medials}

$\S 4$. Associated with four medials there is a bilinear concomitant

$$
\Phi_{1}=\left(u a^{\circ}\right)\left(A^{\prime \cdot} B c^{\prime}\right)\left(C^{\prime:} D \xi\right), \quad a A^{\prime}=A, c C^{\prime}=C^{\prime}
$$

where $\xi$ denotes a variable point and $u$ a variable prime. This is a matrix outer product $u A B C D \xi$, with $\sigma=0, n=2 k$.

Clearly $\xi, C D \xi, A B C D \xi$ respectively, represent three points $\xi, z, x$ say, where $z$ is in $C$ and $x$ in $A$. We have $z=C D \xi, x=A B z$, which shew, by $\S 3(4)$, that a unique line through a general point $\xi$ of [2k-1] meets both $C$ and $D$, meeting. $C$ at $z$, and again a unique line through $z$ meets both $A$ and $B$, meeting $A$ at $x$. We assume that $A, B, C, D$ are in general position. As $z$ ranges over $C, x$ ranges over $A$.

Thus $\Phi_{1}$ gives a point to point collineation between points $\xi$ of 
[2k-1] and $x$ of $A$, where $x$ is given linearly in terms of $\xi$. Take therefore

$$
\Phi_{1}=u \Phi \xi=\sum_{p, q=1}^{2 f} u_{p} \phi_{p q} \xi_{q}, \quad x_{p}=\sum_{q=1}^{2 k} \phi_{p q} \xi_{q}
$$

introducing a $2 k \times 2 k$ matrix $\left[\phi_{p q}\right]$, where the element $\phi_{p q}$ is the coefficient of $u_{p} \xi_{q}$ in the expression (1).

Since $\Phi_{1}$ transforms the whole space $[2 k-1]$ to a medial $[k-1]$, this matrix $\Phi$ is singular, and of rank $k$ at most.

The absolute bilinear form of contragredience $u \xi=\sum_{p=1}^{2 k} u_{p} \xi_{p}$ corresponds to the $2 k \times 2 k$ unit matrix, and to the identical collineation within the space $[2 k-1]$.

The sum of the coefficients of $u_{p} \xi_{p}$ in (1) is the trace $T_{1}$ of the matrix $\Phi$. This yields at once

$$
T_{1}=\left(A^{\prime \prime} B C D a^{*}\right)=X_{k-1} \text {. }
$$

When $A, B, C, D$ are in general position the singular matrix $\Phi$ has a $k \times k$ matrix $M$ for its non-singular core. To prove this we note that the latent points of $\Phi$ occur whenever $x$ and $\xi$ coincide. Such points can only lie with $x$ in the medial $A$. Also when $\xi$ is any point of $A$ it gives a unique $x$ of $A$ as before; but now the process may be reversed, and from a given $x$ a unique $\xi$ of $A$ can be found. Hence a $k \times k$ non-singular matrix $M$ exists, such that $x=M \xi, \xi=M^{-1} x$, where each of $x$ and $\xi$ is expressed by $k$ (not $2 k$ ) components, suitable for points within $A$.

Also when $x$ coincides with $\xi$, a transversal line through $x$ of $A$ cuts all four medials, as the construction at once shews. There are therefore at most $k$ such transversal lines of four medials in general position of $[2 k-1]$; and they will occur when the latent roots of $M$, $k$ in number, are distinct and non-zero, answering to the $k$ latent points.

Since we are dealing with homogeneous coordinates, the same point $x$ is obtained on multiplying by the invariant $(A B)(C D)$. The collineation is now

$$
(A B)(C D) x=A B C D \xi=a_{p}^{\cdot}\left(A^{p \cdot B C D \xi}\right)
$$

where $a_{p} A^{p}=A=a_{1} a_{2} \ldots a_{k}$, and $p$ is any suffix $1,2, \ldots, k$, and $A^{p}$ denotes the $k-1 a^{\prime}$ s distinct from $a_{p}$. Let this be called the normalized form of the collineation.

But since $\xi$ is assumed to be a point of $A$ it can be given by

$$
\xi=a_{1} \theta_{1}+\ldots+a_{k} \theta_{k}
$$


in terms of the $k$ points $a_{i}$ and scalar components $\theta_{i}$. Similarly let $x=\Sigma a_{p} \theta_{p}^{\prime}$. From (4) we get $(A B)(C D) \theta_{p}^{\prime}=\left(A^{p} B C D \xi\right)$. This gives the collineation $x \rightarrow \xi$, for points within $A$, in the form

$$
\theta^{\prime}=N \theta, \quad \quad N=\left[n_{p q}\right], \quad(A B)(C D) n_{p q}=\left(A^{p} B C D\left(\iota_{q}\right),\right.
$$

where both $p$ and $q$ range over $1,2, \ldots, k$. Thus we have an explicit form for $N$ the normalized matrix. The corresponding matrix $M$ is then

$$
M=\left[\left(A^{p} B C D a_{q}\right)\right] \text {. }
$$

The latent roots $\mu_{1}, \ldots, \mu_{k}$ of $N$ are given by the characteristic equation

$$
|M-\mu(A B)(C D) I|=0 \text {. }
$$

For a latent point we have $\theta^{\prime}=\mu_{i} \theta$, that is

$$
\sum_{q}\left(A^{p} B C D a_{q}\right) \theta_{q}=\mu_{i}(A B)(C D) \theta_{p}, \quad p=1,2, \ldots, k .
$$

\section{Compound Collineations}

$\S 5$. From two points $\xi, \eta$ we can derive image points $x, y$ both in $A$, such that $x=A B C D \xi, y=A B C D \eta$. This at once causes the line $x y$ to be the image of the line $\xi \eta$, The line coordinates $x y$ are then given linearly in terms of the line coordinates $\xi \eta$ by the second compound of the matrix $\Phi$. Similarly from $l$ points $\xi, \eta, \ldots$ we derive an $[l-1]$, say $\Xi_{l}$, which has an image $[l-1]$ given by $x y \ldots$, obtained by the $l^{\text {th }}$ compound of the matrix $\Phi$.

Now we may denote these compound collineations by the expressions

$$
\Phi_{l}=\left(U_{l} A_{l}\right)\left(A_{i}^{*} B C_{l}^{:}\right)\left(C_{i}^{:} D \Xi_{l}\right)=(U A B C D \Xi),
$$

where $i+l=k, n=2 k, l=1,2,3, \ldots, k-1$. For completeness we also define the case $l=0$ as

$$
\Phi_{0}=(A B)(C D) \text {. }
$$

In proof of (1) we consider the three products $\Xi_{l}, C D \Xi_{l}, A B C D \Xi_{l}$, each of which denotes an $[l-1]$. By $\S 4$ if $\xi$ is any point of $\Xi_{l}$, the second product gives that point $z$ of $C$ where the line transversal of $C, D, \Xi_{l}$ meets $C$ : and as $\xi$ ranges over $\Xi_{l}, z$ ranges over $\Xi_{c}\left(=C D \Xi_{l}\right.$ ). Similarly the third product gives the image of $\Xi_{l}$ in $A$. Thus the expression ( 1 ) denotes the $l^{\text {th }}$ compound.

The matrix $\Phi^{(l)}$ obtained by deleting the variables $U$ and $E$ in (1) will have $\left(\begin{array}{c}2 k \\ l\end{array}\right)$ rows and columns. To a scalar factor it must there- 
fore be the $l^{\text {th }}$ compound of the matrix $\Phi$. The trace of this new matrix is given by the sum of its principal diagonal elements; and this sum is obtained at once from (1) by deleting $U$ and $\Xi$, and then permuting $A_{l}$ with $C_{i} D$, getting $\left(C_{i} D A_{l}\right)$. This gives, for the trace,

$$
T_{l}=\left(A_{i}^{*} B C_{l}^{i}\right)\left(C_{i}^{\vdots} D A_{l}\right)=X_{i}
$$

which is the invariant already discussed.

The precise value of the $l^{\text {th }}$ compound matrix $\Phi^{(l)}$ of $(1)$ is given by the formula

$$
M^{(l)}=\Phi_{0}^{l-1} \Phi^{(l)}
$$

where $M^{(l)}$ is the $l^{\text {th }}$ compound of the matrix $M$, and $\Phi_{0}$ is the scalar factor $(A B)(C D)$. For $M$ is the matrix of the bilinear form $\Phi_{1}(\xi)$ given in $\S 4(1)$, and $M^{(2)}$ is that of $\Phi_{1}\left(\xi^{*}\right) \Phi_{1}\left(\eta^{*}\right) \equiv \Phi_{2}(\xi \eta)$, say; and again $M^{(3)}$ is that of $\Phi_{2}\left(\xi^{*} \eta^{\circ}\right) \Phi_{1}\left(\zeta^{\circ}\right)$; and so on. Now consider $\Phi_{l}\left(\Xi_{l}\right) \Phi_{1}(\xi)$ where the $l+1$ points $\Xi \xi$ are to be permuted. We have

$$
\begin{aligned}
& \left(U_{l} A_{l}\right)\left(A_{i} B C_{l}\right)\left(C_{i} D \Xi_{l}^{*}\right)(u a)\left(A^{\prime} B c^{\circ}\right)\left(C^{\prime} D x^{\circ}\right) \\
= & \left(U_{l} A_{l}\right)\left(A_{i} B C_{l}\right)\left(C_{i-1}^{\circ} x D \Xi\right)(u a)\left(A^{\prime} B c^{\circ}\right)\left(C^{\prime} D c^{\circ}\right)
\end{aligned}
$$

where $C=C_{i} C_{l}=C_{i-1} c C_{l}=c C^{\prime}$. The last factor, having $k$ symbols $c$, can only be $(C D)$ or else zero. This gives $\left(A^{\prime} B c^{\circ}\right)\left(C^{\prime} D c\right)$ for the two last factors. Hence, if $C_{l} C_{i}$ are also convolved in the original expression we have

$$
\left(U_{l} A_{l}\right)\left(A_{i} B C_{l}\right)\left(C_{i-1}^{*} x D \Xi_{l}\right)(u a)\left(A^{\prime} B c^{\circ}\right)\left(C^{\prime} D c\right) .
$$

On convolving $C_{i} c$ in the second factor we at once get $\left(A^{\prime} B a\right)\left(C^{\prime} D c\right)$ for the final factors. And when the $a$ 's are permuted in two sets of $k$ this gives

$$
\begin{aligned}
& \left(U_{l} A_{l}^{*}\right)\left(A_{i-1}^{*} c^{*} B C_{l}^{*}\right)\left(C_{i-1}^{:} x D \Xi\right)\left(u a^{\dot{*}}\right)(A B)(C D) \\
& =\left(u U \dot{a_{A} \dot{A}_{l}}\right)\left(\dot{A_{i-1}} c^{*} B C_{l}^{*}\right)\left(C_{i-1}^{:} x D \Xi\right) \Phi_{0} \\
& =\left(U_{l+1} \dot{A_{l+1}}\right)\left(\dot{A_{i-1}} B C_{l+1}^{i}\right)\left(C_{i-1}^{i} D \Xi_{l+1}\right) \Phi_{0} \\
& =\Phi_{i+1} \Phi_{0} \text {. }
\end{aligned}
$$

Hence the factor $\Phi_{0}$ enters at each step in passing from one compound to the next, so that (4) follows by induction.

Again the traces of the compounds $M^{(i)}$ are well known to be the coefficients of powers of $\lambda$ in the characteristic function $|M-\lambda I|$ of $M$. Hence by (3) and (4) the characteristic equation of $M$ can be written

$\lambda^{k}-\left(A_{k-1}^{\cdot} B C D a^{\circ}\right) \lambda^{k-1}+(A B)(C D)\left(A_{k-2}^{*} B C D A_{2}^{*}\right) \lambda^{k-2}-\ldots=0$

and that of $|N-\mu I|=0$ can be written

$$
X_{k} \mu^{k}-X_{k-\mathrm{J}} \mu^{k-1}+X_{k-\mathfrak{q} \mu^{k-2}}-\ldots+(-)^{k} X_{0}=0 .
$$


Hence the $k+1$ quadrilinear invariants $X$ are the coefficients of the characteristic equation for the collineation $x=A B C D \xi / \Phi_{0}$ when taken in normalized form.

The latent roots $\mu_{i}$ can be identified with cross ratios $\{A B C D\}_{i}$ in which the four medials cut their $k$ common transversal lines, in the following way. If $\phi, \psi, \chi$ denote sets of $k$ coordinates for a point within $B, C, D$ respectively, then the relations

$$
A \theta^{\prime}+B \phi+C \psi=0, A \theta+C \psi+D_{\chi}=0
$$

hold when, and only when, the points $\theta^{\prime} \phi \psi$ are collinear as also the points $\theta \psi \chi$. But these are precisely the geometrical conditions of the collineation $\theta^{\prime}=N \theta$ within $A$. Now consider the latent point (when $A \theta$ coincides with the point $A \theta^{\prime}$ ), corresponding to the latent root $\mu_{i}$. Then $\theta^{\prime}=\mu_{i} \theta$ and the lines $\theta^{\prime} \phi \psi, \theta \psi \chi$ coincide and give four collinear points, one on each medial,

$$
A \theta, \quad A \theta+C \psi, \quad C \psi, \quad \mu_{i} A \theta+C \psi,
$$

or simply $a, a+c, c, \mu a+c$, in terms of the points $a=A \theta, c=C \psi$. The cross ratio of these points is $\mu_{i}$. Hence the latent root of the collineation is equal to the cross ratio $\{A B C D\}$ say, of the four collinear points where a transversal cuts the medials: and there are generally $k$ such transversals, one for each root and cross ratio.

We must note that the matrix $N$, whose latent roots give these cross ratios, has been normalized, in the sense that each of its elements is an absolute invariant of the points of the four medials, owing to the introduction of their common denominator $(A B)(C D)$. Geometrically the same collineation $\Phi_{1}$ is given by any scalar multiple of $N$, so that only the ratios of the cross ratios are given if the point collineation of $A$ is given.

The existence of the three sets of invariants $X, Y, Z$ of four medials, and the cyclic relations between them, now fall into line with the well-known permutative properties of cross ratios. On writing the cross ratios of four points in the forms

$$
\{A B C D\}=\mu, \quad\{A D B C\}=\mu^{\prime}, \quad\{A C D B\}=\mu^{\prime \prime},
$$

where $\mu^{\prime}=1 /(1-\mu), \mu^{\prime \prime}=1-1 / \mu$, then the identities (11) of $\S 1$ at once shew that the characteristic equation (7) for $\mu$ in terms of the $X_{i}$ is capable of two further modes-for $\mu^{\prime}, \mu^{\prime \prime}$ in terms of the $Z_{i}, Y_{i}$ respectively.

The six permutations of $B, C, D$ consequently give rise to six collineations within $A$, changing a point $x$ to $\xi$ or to $\xi^{\prime}$ or to $\xi^{\prime \prime}$, and 
reciprocally $\xi$ or $\xi^{\prime}$ or $\xi^{\prime \prime}$ to $x$. All six collineations evidently have the same latent points, $k$ in number; and their six sets of latent roots correspond to the six sets of cross ratios $\mu_{i}, \mu_{i}^{\prime}, \mu_{i}^{\prime \prime}, \mu_{i}^{-1}$, etc.

Thus there are three characteristic equations of order $k$, with coefficients $X_{i}, Y_{i}, Z_{i}$ respectively, for a set of four medials: and their three sets of roots correspond to the three pairs $\mu, \mu^{-1}$ of cross ratios determined by four collinear points. If $\mu_{i}$ is a root of the $X$ equation then those of $Y$ and $Z$ are $\left(\mu_{i}-1\right) / \mu_{i}$ and $\left(1-\mu_{i}\right)^{-1}$ respectively.

Interesting particular results follow. If $\mu_{i}=-1$ then the transversal is cut harmonjcally by the pairs $A C, B D$. Hence

$$
X_{0}+X_{1}+\ldots+X_{k}=0
$$

is the necessary and sufficient condition for the medials $A C$ to separate harmonically the medials $B D$ upon one of the $k$ transversal lines of all four medials in [2k-1].

Two of the ranges have equal cross ratios if the discriminant of the characteristic $k$ - ic of $X$ or $Y$ or $Z$ vanishes.

When all the roots are equal the four medials belong to the same system of $\propto^{1}$ generators of a certain locus $\mathcal{R}$ of the type $V_{k}^{k}$, described by the $\infty^{k}$ straight lines which meet all four medials. The collineation becomes the identical transformation, and the matrix $M$ (or $N$ ) is scalar.

\section{The quaternary case}

$\S 6$. Here $k=2$ and, let us say,

$$
\begin{aligned}
& X_{0}=(B C)(D A)=Y_{2}, \\
& Y_{0}=(C A)(B D)=Z_{2}, \\
& Z_{0}=(A B)(C D)=X_{2} .
\end{aligned}
$$

Also $X_{1}=\left(a^{\circ} B c^{\prime}\right)\left(c^{\prime} D a^{\prime *}\right)$, etc. The conditions $\S 1$ (9) reduce to

$$
X_{1}=X_{0}-Y_{0}+Z_{0}, \quad Y_{1}=X_{0}+Y_{0}-Z_{0}, \quad Z_{1}=-X_{0}+Y_{0}+Z_{0} \text {. }
$$

The quadratic equation

$$
X_{0}-X_{1} \mu+X_{2} \mu^{2}=0
$$

gives the cross ratios of the transversals (now two in number) of the four lines $A, B, C, D$ in [3]. The condition for $A C$ to separate $B D$ harmonically upon one transversal is $X_{0}+X_{1}+X_{2}=0$, which reduces to

$$
2(B C)(D A)-(C A)(B D)+2(A B)(C D)=0
$$


The two cross ratios are equal when $X_{1}^{2}=4 X_{0} X_{2}$, which gives

$$
\sqrt{ } X_{0}+\sqrt{ } Y_{0}+\sqrt{ } Z_{0}=0,
$$

a well-known result. This happens when any one line $D$ touches the quadric through the other three lines, so making the two transversals coincide; in particular when $D$ is a generator of either system on this quadric surface.

\section{Canonical Forms}

$\S 7$. On taking $A$ and $C$ to be the frame of reference, with $[A, C]$ as a $2 k \times 2 k$ unit matrix, we can express any four skew medials in the form

$$
A=\left[\begin{array}{l}
I \\
0
\end{array}\right], \quad B=\left[\begin{array}{l}
I \\
I
\end{array}\right], \quad C=\left[\begin{array}{l}
0 \\
I
\end{array}\right], \quad D=\left[\begin{array}{l}
I \\
N
\end{array}\right],
$$

where $I$ is the $k \times k$ unit matrix, and $N$ is at present a general $k \times k$ matrix. For, with these values of $A$ and $C$, this is the form which $B$ and $D$ assume when the parameters, in the conditions $A \theta^{\prime}+B \phi+C \psi=0$ and $A \theta+C \psi+D \chi=0$, are taken to satisfy $\theta^{\prime}=-\phi=\psi=-\chi$, while $\theta^{\prime}=N \theta$. This is legitimate since the choice of frame of reference within each medial is arbitrary.

The three points $a_{i}, b_{i}, c_{i}$ of $A, B, C$ respectively are now collinear, for $i=1,2, \ldots, k$ : and if $a_{i}$ is a latent point this line also contains the point $d_{i}$ of $D$. When the collineation $\Phi_{1}$ has $k$ latent points, we may take them to be these $a_{i}$, for which $\theta^{\prime}=\mu_{i} \theta$, so that

$$
N=\operatorname{diag}\left(\mu_{1}, \mu_{2}, \ldots, \mu_{k}\right) \text {. }
$$

The invariants $X_{i}=\left(A_{i}^{\circ} B C D A_{l}\right)$ can easily be calculated for these values of $A, B, C, D$ in (1) and (2). The result is

$$
X_{0}=(-)^{k} \mu_{1} \mu_{2} \ldots \mu_{k}, X_{i}=(-)^{k} \Sigma \mu_{p_{1}} \mu_{p_{2}} \ldots \mu_{p_{l}}, X_{k}=(-1)^{k} \text {. }
$$

These $X_{i}$ are in fact the elementary symmetric functions of the latent roots of $N$, and this gives another proof that the characteristic equation , $N-\mu I \mid=0$ has the $X_{i}$ for its coefficients. But the earlier proof is more general, since it would apply to any $N$, with or without repeated latent roots.

The original collineation $u \Phi \xi=(u A B C D \xi)$, which has a singular $2 k \times 2 k$ matrix, can also be calculated in this case (1) above. It gives

$$
\Phi=\left[\begin{array}{cc}
N & I \\
0 & 0
\end{array}\right]
$$


For example, when $k=3$, and $N$ is in canonical form (2),

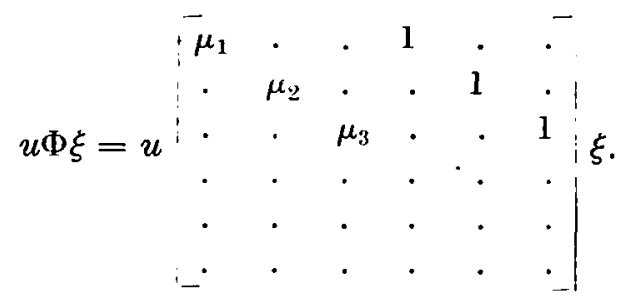

Manifestly the characteristic equation for $\Phi$ is

$$
\left(\mu-\mu_{1}\right) \ldots\left(\mu-\mu_{k}\right) \mu^{k}=0
$$

and the traces of $\Phi$ and all its compounds are the same as those of $N$. The reduced characteristic equation of $\Phi$ is the $(k+1)$-ic $\mu \Pi\left(\mu-\mu_{i}\right)=0$.

If $A=a_{1} A^{2}=a_{i} A^{i}, i \neq 1$, and $a_{1}$ is a latent point then $\left(A^{i} B C D a_{1}\right)=0, \quad\left(A^{1} B C D a_{1}\right)=\mu_{1}(A B)(C D)$ as follows from the canonical form of $\S 6$ (4). Similarly $a_{1} a_{2}$ is a latent line if $\left(A^{i j} B C D a_{1} a_{2}\right)=0$ or $\mu_{1} \mu_{2}(A B)(C D)$, according as $i, j \neq$ or $=1,2$, where $A=a_{i} a_{j} A^{i j}$. And so on. These are the necessary and sufficient invariant conditions for latency of particular spaces within $A$. Here $B, C, D$ can be taken in general coordinates.

The vanishing of $X_{i}$

$\S 8$. On writing $A_{l}^{\prime}$ for $B C D A_{l}$, the image of $A_{l}$ in the collineation, we can express the invariant $X_{i}$ as a sum of determinants $\left(A_{i} A_{i}^{\prime}\right)$, obtained as before by permutation of the $k$ points $a_{1}, \ldots, a_{k}$. When these base points are so chosen that $a_{2}$ is the image of $a_{1}, a_{3}$ of $a_{2}$, and so on till $a_{k}$ is reached, then all the terms of $X_{i}$ vanish except one, that in fact for which $A_{i}=a_{1} a_{2} \ldots a_{i}$. This is because $A_{i}$ and $A_{i}$ contain at least one $a_{r}$ in common for every other term.

For example, if $k=4, X_{2}=\Sigma(12,34)=\Sigma\left(123^{\prime} 4^{\prime}\right)$ by an obvious notation; and $1^{\prime}=2,2^{\prime}=3,3^{\prime}=4$ for the image points. The term $\left(123^{\prime} 4^{\prime}\right)=\left(1244^{\prime}\right) \neq 0$, whereas any one of the other five terms vanishes $\left(\left(132^{\prime} 4^{\prime}\right)=\left(1334^{\prime}\right)=0\right)$.

The last point $a_{k}$ has for its image a linear combination of the earlier $k-1$ points, say

$$
a_{k+1}=a_{k}^{\prime}=\sum_{m=1}^{i-1} a_{m} a_{m}
$$

where the coefficient $\alpha_{m}$ is $(-)^{m+1} X_{m} / X_{k}$, since these values would 
give the requisite characteristic equation, on taking the $k$ points $a_{r}$ to be $\theta, N \theta, \ldots, N^{*-1} \theta$ respectively ${ }^{1}$.

Accordingly, if $X_{i}$ vanishes, the space $A_{i}$ meets $A_{l}^{\prime}$, where $A_{l}^{\prime}$ is the image of a space $A_{l}$ complementary to $A_{i}$ in the simplex defining the whole space $A$. This is a result due to Segre $^{2}$.

Alternatively we can state the condition thus: $X_{i}$ vanishes when the image of the $k^{\text {th }}$ and final independent point of the chain $a_{1} a_{2} \ldots$ lies in $a[k-2]$ through all but the point $a_{i+1}$ of these $k$ points.

For we have by construction the relation

$$
X_{0} a_{1}-X_{1} a_{2}+\ldots+(-)^{k} X_{k} a_{k+1}=0
$$

which gives $a_{k+1}=a_{k}^{\prime}$ as the first point of the chain to depend on previous points, where the coefficients in such a relation are necessarily those of the characteristic equation. Hence $a_{k+1}$ cannot depend on $a_{i+1}$ if $X_{i}=0$.

The above conditions are necessary and sufficient for the case when the characteristic equation cannot be reduced to one of lower degree, in fact for the case of one invariant factor. Segre shewed that the above geometrical condition suffices to cause $X_{i}$ to vanish in all cases, and is necessary also in the four cases when $i=1,2, k-2$, $k-1$. For the case of two or more invariant factors the above set $a_{1}, \ldots, a_{k}$ no longer holds, and (1) is replaced by a relation of lower degree. It is easy to express $X_{i}$ in terms of a corresponding (and more complicated) basis, but the geometrical statement is more elaborate.

\section{Extensionals to even dimensions}

$\S 9$. Corresponding to $X_{i}$ in [2k-1] there is a quadric primal of [2k] given by $(x A B C D x)_{i}=0$, where $x$ has $2 k+1$ elements, and each of $A, B, C, D$ denote $k$ columns each of $2 k+1$ elements. Such a quadric is the locus of a point $x$ in [2k] through which four [k]'s $x A, x B, x C, x D$ are drawn to cut an arbitrary prime [2k-1], not containing $x$, in four [ $k-1]$ 's whose invariant $X_{i}$ vanishes.

More interesting is the harmonic quadric primal of four $[k-1]$ 's in $[2 k]$, namely the locus

$$
\sum_{i=1}^{k}(x A B C D x)_{i}=0
$$

1 Cf. Turnbull and Aitken, Canonical Matrices (Blackie, 1932), p. 48.

2 Mehrdimensionale Rïume, Encyk. Math. Wissenschaften, III. C 7, 841, where an earlier result by Segre (Math. Annalen, 24 (1884), 152-156), is utilized, which concerns two quadrics. This is relevant since any collineation can be resolved into successive reciprocation in two quadrics by a theorem of Frobenius. 
which is the extensional of $\Sigma X_{i}=0$. This means that $A, B, C, D$ are four arbitrary $[k-1]$ 's in [2k]. They are met by a plane abcd in a single point of each. This plane contains a unique conic through $a b c d$, for which $a$ and $c$ separate $b$ and $d$ harmonically. The locus of points of this conic as the plane takes all possible positions is a quadric primal.

Three such harmonic quadrics exist for the four given $[k-1]$ 's, corresponding to the pairings $a b, c d ; a c, b d ; a d, b c$.

In proof of this take any point $x$ on the conic $a b c d$, and project these points, and the spaces $A, B, C, D$ from $x$ to an arbitrary prime. The result will be four collinear points $a^{\prime} b^{\prime} c^{\prime} d^{\prime}$, one on each of four $[k-1]$ 's in this prime $[2 k-1]$. Also $a^{\prime} b^{\prime} c^{\prime} d^{\prime}$ is a harmonic range, for which $\Sigma X=0$. Hence (1) is true of [2k].

Through an arbitrary point $x$ of $[2 k]$ just $k$ planes can be drawn which meet four skew [k-1]'s, corresponding to the $k$ lines traversing four medials in [2k-1]. Two of the $k$ planes coincide when $x$ satisfies the extensional of the discriminantal equation: that is, the discriminant of the characteristic equation $\Sigma \pm X_{i} \mu^{i}=0$ being formed, $\Delta\left(X_{i}\right)$, say, then the locus of $x$ is $\Delta\left((x A B C D x)_{i}\right)=0$. Since the discriminant is of degree $2(k-1)$ in the coefficients $X_{i}$, each of which corresponds to two of the $x$, the locus of $x$ is of order $4(k-1)$. Hence the locus of a point, through which two of the $k$ possible transversal planes of four [k-1]'s in [2k] coincide, is a primal of order $4(k-1)$.

When $k=2$ this gives Segre's quartic manifold in four dimensions.

The University, ST ANDREWs. 